\title{
Examining the Association Between Parental Socioeconomic Status and Preterm Birth Using Multidomain Social Determinants Scale in a Tertiary Care Center in Saudi Arabia
}

\author{
Mohammed Y. Al-Hindi ${ }^{1,2,} 3$, Hazem Aljuhani ${ }^{2}$, Anas R. Alnajjar ${ }^{2}$, Sarah Alessa ${ }^{4}$, Mansour Alqurashi ${ }^{1,2}$, \\ 3 , Yaser A. Faden $5,2,3$ \\ 1. Pediatrics, King Abdulaziz Medical City - Western Region, Ministry of National Guard, Jeddah, SAU 2. College of \\ Medicine, King Saud Bin Abdulaziz University for Health Sciences, Jeddah, SAU 3. Research and Development, King \\ Abdullah International Medical Research Center - Western Region, Jeddah, SAU 4. College of Medicine, King Abdulaziz \\ University Hospital, Jeddah, SAU 5. Obstetrics and Gynecology, King Abdulaziz Medical City - Western Region, \\ Ministry of National Guard, Jeddah, SAU
}

Corresponding author: Mohammed Y. Al-Hindi, alhindimo@ngha.med.sa

\section{Abstract \\ Objectives}

Socioeconomic status (SES) plays a conflicting role in preterm birth (PB). This study evaluated the association between SES and PB using, for the first time, a multidomain scale, validated for Saudi Arabia, with a scoring system and examined the effect of each SES domain on PB. The secondary outcome was to determine the effects of SES on birth weight (BW) and the subcategories of PB and BW.

\section{Methods}

This cross-sectional study was conducted between May 2017 and August 2017 at a National Guard tertiary center in Jeddah, Saudi Arabia. A total of 477 parents were interviewed using the Elzahrany R. SES scale.

\section{Results}

The rate of PB was 11.5\%, with no significant differences among the high, middle, and low SES classes (13\%, $11 \%$, and $12.5 \%$, respectively). There were no patients in the very low SES in this specific population. None of the maternal or neonatal characteristics were significantly different among SES classes except maternal age $(\mathrm{p}$ value $=0.03)$, and antenatal care recorded visits "booking" status ( $\mathrm{p}$ value $=0.012$ ). Stratified analysis for PB subcategories showed the lower SES classes had higher moderate (3.8\%) and extreme (1.6\%) PB. For BW subcategories, large for gestational age (LGA) infants were higher in the high SES class (13\%). However, the lower SES classes had higher rates of lower BW. The association between SES and PB remained not significant after adjusting for the maternal age and antenatal booking status.

Received 07/30/2020

Review began $07 / 31 / 2020$ Review ended 09/13/2020 Published 09/17/2020

() Copyright 2020 Al-Hindi et al. This is an open access article distributed under the terms of the Creative Commons Attribution License CC-BY 4.0., which permits unrestricted use, distribution, and reproduction in any medium, provided the original author and source are credited.

\section{Conclusion}

There was no association between SES and PB at a tertiary center providing universal care to the National Guard using multidomain socioeconomic determinants with a scoring system. However, lower SES was associated with lower BW. The use of the "polysocial risk score" based on locally validated surveys should be considered in any health research that examines the effects of socioeconomic determinants.

Categories: Obstetrics/Gynecology, Pediatrics, Public Health

Keywords: multidomain scale, preterm delivery, socioeconomic status, polysocial risk score, preterm birth, birth weight, socioeconomic factors

\section{Introduction}

Preterm birth (PB), i.e., birth before 37 weeks of gestation, is a significant burden worldwide as it is the second direct cause of neonatal mortality. In a year, 15 million neonates are born preterm, and the incidence is higher in lower income countries than in high-income ones [1]. Approximately half of the PBs are idiopathic [2].

Recently, several studies revealed the significant role of socioeconomic status (SES) on different aspects of health, including physical and psychological, among all age groups in society [3]. SES is a reflective measurement of an individual's positions in a community based on different factors such as education, income, and occupation. According to these factors, individuals are classified into social hierarchy [4]. Previous studies were conducted to investigate the effect of SES on prematurity. Some of these studies showed an association, while others did not [5-7]. These contrasting results may be explained by the 
Researchers have built several scales to include various factors that are determined by differences in culture and geographical location. In the United States and India, the most commonly used scales are Hollingshead and Kuppuswamy scales, respectively [8,9]. Moreover, the Fahmy and El-Sherbini and El-Shakhs (The scale manual. El-Shakhs AE. A scale of socioeconomic status of the family. Cairo, Anglo-Egyptian Stationery, 1995) scales are the only scales in the Middle East [10]. The former is generally used in health research, while the latter is in educational fields [11]. The updated and validated Fahmy and El-Sherbini scale categorizes families into five classes based on seven domains [11]. Elzahrany, a sociologist, formulated and validated a multidomain, socioeconomic determinant with a scoring system in Saudi Arabia, which consists of seven domains adopted from the Fahmy and El-Sherbini scale (Unpublished research. Elzahrany RA: Socioeconomic Levels and Standards of Living in Makkah “Mecca”, Saudi Arabia. Geography Department, College of Social Sciences, Umm Al-Qura University, 2017).

To the best of our knowledge, no published study in Saudi Arabia has measured SES based on a scale and scoring system. Moreover, the association between SES and prematurity in the region has not yet been elucidated. Thus, this study aims to evaluate the association between SES and PB using a validated multidomain scale (Elzahrany R. scale) with a scoring system validated for Saudi Arabia. The secondary objectives are to examine the effect of each SES domain on PB and determine the low-birth-weight (LBW) distribution among SES classes.

\section{Materials And Methods}

This cross-sectional study was conducted in pre- and postnatal care units and neonatal intensive care unit (NICU) of King Abdulaziz Medical City (KAMC), Ministry of National Guard, Western Region, Jeddah, Saudi Arabia. The survey period was between May 2017 and August 2017. The study followed the principles of the Helsinki Declaration, and Institutional Review Board (IRB) approval was obtained from the IRB of King Abdulla International Medical Research Center. The study participants included all parents of newborns in these care units. On the other hand, parents who refused to participate, had multiple pregnancies (more than two kids), or had newborns diagnosed with severe/chromosomal congenital anomalies were excluded from the study.

A sample size of 477 was calculated based on a population data that calculated the prematurity rate $11.7 \%$ in unit audit in 2015, an estimated incidence $6.5 \%$ of prematurity rate in the lower SES, a level of significance of $5 \%$, and a power of $80 \%$ to detect a statistically significant difference.

A non-probability consecutive sampling technique was used in which all included were identified from the admission logbooks in the expected wards. The participants were categorized into "case," those who had PB, and control groups, those with term ( $\geqslant 37$ weeks of gestation) birth. After obtaining consent, the primary household was interviewed directly or by telephone for the socioeconomic determinant data. Subsequently, we collected the parental and neonatal basic demographics in addition to the designation of newborns from hospital health electronic records. PB was subcategorized into late (LPB, birth between 34 and 36 weeks), moderate (MPB, birth between 28 and 33 weeks), and extreme (EPB, birth between 23 and 27 weeks). Birth weight (BW) was subcategorized into large (LBW, birth weight > 4,000), normal (birth weight of 2,500-4,000 g), low (LBW, birth weight of 2,500-1,500 g) very low (VLBW, birth weight of 1,000-1,499 g), and extreme (ELBW, birth weight < 1,000 g).

The questionnaire developed by Elzahrany was used to gather the socioeconomic details. It aimed to measure SES via a validated scoring system that fits Saudi Arabia. Elzahrany's team was composed of a sociologist and epidemiologist and medical professionals experienced in the field and social studies. This scale also consists of four main domains, which were adopted from the Fahmy and El-Sherbini scale [11]: (1) residential characteristics; (2) demographics that included four sub-domains: the number of family members, education, occupation, and income of both parents; (3) tourism and entertainment; and (4) health status of family members. This questionnaire was collected via two separate field surveys conducted in 2016 and 2017 in which the participants were selected via a random cluster sample and the results were validated through kappa statistics, which showed strong agreement. Factor analysis was used to calculate the scores. We did not need to validate language as both surveys, Elzahrani R. and Fahmy, were in Arabic. SES was then categorized into four classes (high, middle, low, and very low) based on the 25th segment increments of the participant's scores (see attached Appendix).

Data were collected via a Google form that generated an Excel sheet of raw data and were screened for any missing data and outliers. Quantitative data were described by central tendency (mean and standard deviation [SD], median and interquartile range [IQR]) and graphs (histogram and box plots), whereas frequency tables and graphs (bar charts) were for qualitative data. The student's t-test or the median test was performed to compare quantitative data accordingly. On the other hand, chi-square/Fisher's exact test was used for the comparisons of qualitative data. A p value of $<0.05$ was considered significant. Data were analyzed using the IBM ${ }^{\circledR}$ SPSS ${ }^{\circledR}$ Version 24 (Armonk, NY, IBM Corp.). Stratified and logistic regression analyses were planned to assess confounding factors. 


\section{Cureus}

\section{Results}

A total of 987 participants were recruited in the study. Among these, only 485 (49\%) responded, of which 33 (6.8\%) refused to be included and 10 were excluded as per the exclusion criteria. Therefore, we interviewed 442 parents. The mean SES score was 55.4 ( $($ SD 10) and a median of 54 (50\% IQR 49-61). There were 120 participants (27.1\%) who were scored between 25 and 49 (low SES status level) with a median of 46 (IQR 4348). On the other hand, 299 (76.5\%) subjects were scored between 50 and 74 (middle SES level) with a median of 56 (IQR 53-62). Only 23 (5.2\%) participants were scored 75 or more (high SES level) with a median of 78 (50\% IQR 76-79). Finally, no participants were scored < 25 (very low SES class); therefore, this class was not analyzed.

In maternal demographics, the mean age of the mothers was $31.4 \pm 5.4$ years in the high class compared with $28.2 \pm 5.3$ and $28.4 \pm 6.4$ in the middle and low classes, respectively ( $p$ value $=0.033$ ). Moreover, the number of booked mothers (pregnant with a recorded regular antenatal follow-up) was higher in the middle class with a percentage of $84.9 \%$ compared with $77.3 \%$ and $72.5 \%$ in the high and low classes, respectively ( $\mathrm{p}$ value $=0.012$ ). The remaining maternal demographics showed no statistically significant differences were noted among the classes. The pregnancy-related disease was more common in the high class but was not statistically significant. In neonatal demographics, BW, gestational age, cord pH, and Apgar score were not statistically significantly different. There was a trend toward less small for gestation and admission to NICU in the high class, although it was not statistically significant (Table 1).

\begin{tabular}{|c|c|c|c|c|}
\hline Characteristics & Low class $(n=120)$ & Middle class ( $n=299$ ) & High class $(n=23)$ & $p$ values \\
\hline \multicolumn{5}{|l|}{ Maternal } \\
\hline Age $(M \pm S D)$ & $28.4 \pm 6.4$ & $28.2 \pm 5.3$ & $31.4 \pm 5.4$ & 0.033 \\
\hline Height in $\mathrm{cm}(\mathrm{M} \pm \mathrm{SD})$ & $156.1 \pm 5.7$ & $156.9 \pm 6.0$ & $158.8 \pm 5.5$ & 0.110 \\
\hline Weight in $\mathrm{kg}(\mathrm{M} \pm \mathrm{SD})$ & $72.1 \pm 16.8$ & $72.7 \pm 14.3$ & $75.1 \pm 13.9$ & 0.675 \\
\hline Gestational diabetes (\%) & $16.9 \%$ & $12.4 \%$ & $27.3 \%$ & 0.120 \\
\hline Gestational hypertension (\%) & $4.2 \%$ & $5.4 \%$ & $9.1 \%$ & 0.463 \\
\hline Booking status (\% booked) & $72.5 \%$ & $84.9 \%$ & $77.3 \%$ & 0.012 \\
\hline \multicolumn{5}{|l|}{ Neonatal } \\
\hline Gender (\% male) & $42.5 \%$ & $52.2 \%$ & $56.5 \%$ & 0.161 \\
\hline Birth weight in $\mathrm{g}(\mathrm{M} \pm \mathrm{SD})$ & $2879.5 \pm 694.9$ & $3061.2 \pm 1471.9$ & $3277.4 \pm 634.9$ & 0.259 \\
\hline Gestational age $(\mathrm{M} \pm \mathrm{SD})$ & $38.0 \pm 3.2$ & $38.6 \pm 2.2$ & $38.7 \pm 1.6$ & 0.058 \\
\hline Small for gestational age (\%) & $12.5 \%$ & $15.1 \%$ & $4.3 \%$ & 0.318 \\
\hline${ }^{*} \mathrm{Cord} \mathrm{pH}(\mathrm{M} \pm \mathrm{SD})$ & $7.2 \pm 0.08$ & $7.2 \pm 0.08$ & $7.3 \pm 0.07$ & 0.290 \\
\hline Apgar score (median, range) & $9.0,7.0$ & $9.0,10.0$ & $9.0,4.0$ & NA \\
\hline Admission to NICU (\%) & $11.9 \%$ & $7.4 \%$ & $4.3 \%$ & 0.316 \\
\hline
\end{tabular}

TABLE 1: Maternal and neonatal clinical characteristics among SES classes

M, mean; SD, standard deviation

Among the 442 mothers, 391 (88.5\%) had term delivery and 51 (11.5\%) had preterm delivery. There was no difference in SES scores between those who had term or PB [median 54 (50\% IQR 49-61) vs. 55 (50\% IQR 4862)]. The rate of $\mathrm{PB}$ was $11.5 \%$ among all participants, and no significant difference was noted among the SES classes, with PB rates of $12.5 \%, 11 \%$, and $13 \%$ for low, middle, and high SES classes, respectively (p value $=0.9$ ).

In the stratified analysis for further subcategories of PBs, a trend toward an increase in late PB was noted (13\%) in the high SES class compared with low and middle classes. In contrast, lower SES classes had higher moderate and extreme PB (4.2\% and 3.3\%), respectively, however, not statistically significant ( $\mathrm{p}$ value $=0.9$ ). The same analysis for BW categories showed that infants with LBW were higher in the high SES class. However, the lower SES classes had higher rates of VLBW and ELBW (p value $=0.016$ ) (Table 2 ). 


\section{Cureus}

\begin{tabular}{|c|c|c|c|c|}
\hline \multirow{2}{*}{ Categories } & \multicolumn{3}{|l|}{ SES Class } & \multirow[b]{2}{*}{ Total } \\
\hline & Low & Middle & High & \\
\hline \multicolumn{5}{|l|}{ Gestational age, weeks* } \\
\hline Term & 105 (87.5\%) & 266 (89\%) & 20 (87\%) & 391 (88.5\%) \\
\hline Late preterm, 34-36 & $6(5 \%)$ & $25(8.4 \%)$ & $3(13 \%)$ & $34(7.7 \%)$ \\
\hline Moderate preterm, 28-33 & $5(4.2 \%)$ & $7(2.3 \%)$ & $0(0 \%)$ & $12(2.7 \%)$ \\
\hline Extreme preterm, $<28$ & $4(3.3 \%)$ & $1(0.3 \%)$ & $0(0 \%)$ & $5(1.1 \%)$ \\
\hline Total & $120(27.15 \%)$ & 299 (67.65\%) & $23(5.20 \%)$ & $442(100 \%)$ \\
\hline \multicolumn{5}{|l|}{ Birth weight, grams ** } \\
\hline Normal BW & 94 (78.3\%) & $251(84.2 \%)$ & 17 (73.9\%) & $362(82.1 \%)$ \\
\hline Large BW, $>4000$ & $3(2.5 \%)$ & $4(1.3 \%)$ & $3(13 \%)$ & $10(2.3)$ \\
\hline Low BW, 1500-2499 & $15(12.5 \%)$ & 39 (13.1\%) & $3(13 \%)$ & $57(12.9 \%)$ \\
\hline Very low BW, 1000-1499 & $3(2.5 \%)$ & $1(0.3 \%)$ & $0(0 \%)$ & $4(0.9 \%)$ \\
\hline Extremely low BW, <1000 & $5(4.2 \%)$ & $3(1 \%)$ & $0(0 \%)$ & $8(1.8 \%)$ \\
\hline Total & $120(27.21 \%)$ & 298 (67.57\%) & $23(5.22 \%)$ & $441(100 \%)$ \\
\hline
\end{tabular}

\section{TABLE 2: Gestational age and birth weight stratified analysis}

${ }^{*} \mathrm{p}$ value $=0.9$

${ }^{\star \star} \mathrm{p}$ value $=0.016$

We further examined the significant risk factors detected via univariate analysis, namely maternal age and antenatal care booking status. In logistic regression analysis, the association between PB and SES classes was not significant even after adjusting for the included risk factors.

\section{Discussion}

Our study did not find an association between SES and PB. We obtained a prematurity rate of $11.5 \%$ among all deliveries in KAMC, which is similar to the international figures (10.6\%) but higher than the national numbers (3.9\%). This could be explained by the fact that KAMC is considered a tertiary center and therefore accepts higher risk pregnant women.

In stratified analysis, SES was also not associated with PB subclasses (late, intermediate, and extreme). However, BW in the stratified analysis showed that higher SES was associated with LGA. In addition, LBW and VLBW were associated with lower SES classes.

Our study concurs with the Canadian study by Campbell et al. [12]. They examined the effect of the following multiple socioeconomic variables: maternal education, income, immigration prevalence, and minority prevalence on multiple birth adverse outcomes including PB. Socioeconomic data were extracted from the 2011 Canadian Census. None of these socioeconomic variables were significantly associated with PB except for immigration and visible minority prevalence. However, our results are conflicting with those in the study by Parker et al. that used data from the National Maternal and Infant Health Survey by the National Center for Health Statistics [6]. They investigated the effect of multiple socioeconomic domains on PB, LBW, and small for gestational age. Regarding $\mathrm{PB}$, lower maternal and paternal education levels among the black ethnic groups, in addition to certain maternal occupations in both black-and-white ethnic groups, were significantly associated with higher odds of PB. Furthermore, another Italian population-based study by Cantarutti et al. examined the effect of maternal education on several neonatal outcomes [5]. The results showed that a higher maternal education level was significantly associated with reduced odds of PB.

Regarding BW, in the study by Campbell et al., a weak association was noted between maternal education and LBW [12]. However, none of the other socioeconomic variables were associated with LBW. Further analysis was performed using birth percentiles $(<5$ th and $<10$ th) and showed that a higher population density was associated with increased odds of birth of $<10$ th percentile. On the other hand, Parker et al. 
found that lower education levels among black mothers and both black-and-white fathers, in addition to certain maternal occupations in black mothers and low income in both ethnic groups, were significantly associated with higher odds of LBW [6]. This is also consistent with the results in the study by Cantarutti et al. [5]. In our study, we had similar findings as VLBW and ELBW incidences were higher in the low SES class after controlling other biological causes. The high SES class had a higher prevalence of LBW infants, which was not reported in comparative studies $[5,6,12]$. This can be explained by the higher prevalence of gestational diabetes in the high SES class.

All of the above population-based studies showing variable results could be explained by varying reasons. First, different populations with different racial and ethnic backgrounds could contribute to PB. The above examples are of high-income countries. Our study, although local, can highlight the effect of SES on PB on a specific population. This ignites the need for a large population-based national study. Second, the above studies independently examined the effects of different domains of SES. This approach has been recently questioned [13]. Figueroa et al. recommend using the "polysocial risk score" where socioeconomic domains are interlinked and should be taken as a whole rather than as independent segments [13]. To the best of our knowledge, our study is the first that studied the effect of SES on PB using a similar concept with a scoring system that aggregates socioeconomic domains and calculates the final scores.

This is the first national study that examined the effect of SES on PB using a validated multidomain scoring system that reflected our population. It included National Guard soldiers and their families. Those were usually "booked," i.e., followed up regularly in the antenatal clinics. However, approximately $25 \%$ of our population is "not-booked" either due to ineligibility or being accepted as a referral high-risk case, both representing non-military families. Our population was all employed and had universal access to primary and tertiary health-care facilities. Thus, no participants fell under the very low class of SES. Our results are more consistent with those in the Canadian study as both countries have a universal education and health system.

Previous studies discussed the biological pathways leading to PB through neuroendocrine processes precipitated by stressful experiences. These stressful conditions included poor nutrition, prepregnancy health status, lack of medical care, social isolation, and workplace. Our sample did not reflect these stressful living conditions as well as the lack of very low socioeconomic class in the study $[14,15]$.

The limitation of our study is that this is a single-center study reflecting the special population of National Guard soldiers and their families. Hence, our results are reliable but mainly for military/National Guard personnel. However, this could indirectly reflect the effect of a universal health-care system on a targeted population. A low sample size could underestimate the stratified analysis; moreover, the nature of the population did not include ethnic variation data as a factor. Recall bias could affect our results as any survey-based study.

\section{Conclusions}

In conclusion, using a multidomain-validated scoring system to measure SES in Saudi Arabia in a specific population, we did not find an association between SES and PB even with further stratified analysis. However, the SES effect on BW, the stratified analysis showed that the higher SES class was associated with LGA, whereas LBW and VLBW were associated with the lower SES classes. Thus, we recommend conducting a large multicenter cluster random sampling or population-based study to have a better representation and include the very low SES class. Finally, the use of the "polysocial risk score" based on locally validated surveys should be considered in any health research that examines the effects of socioeconomic determinants.

\section{Appendices}

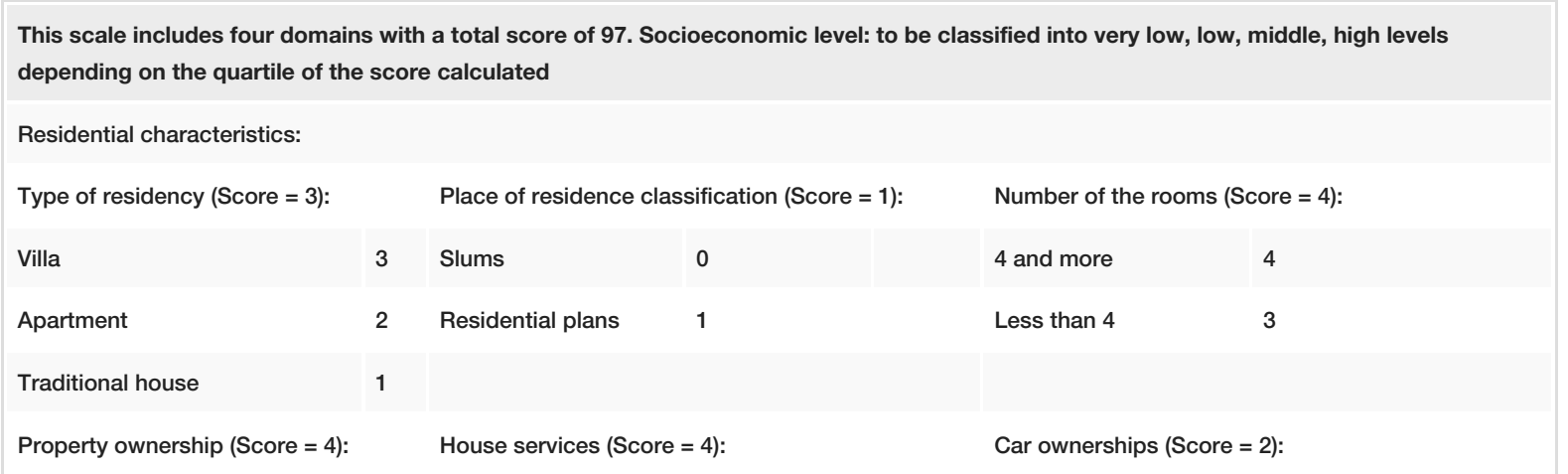




\section{Cureus}

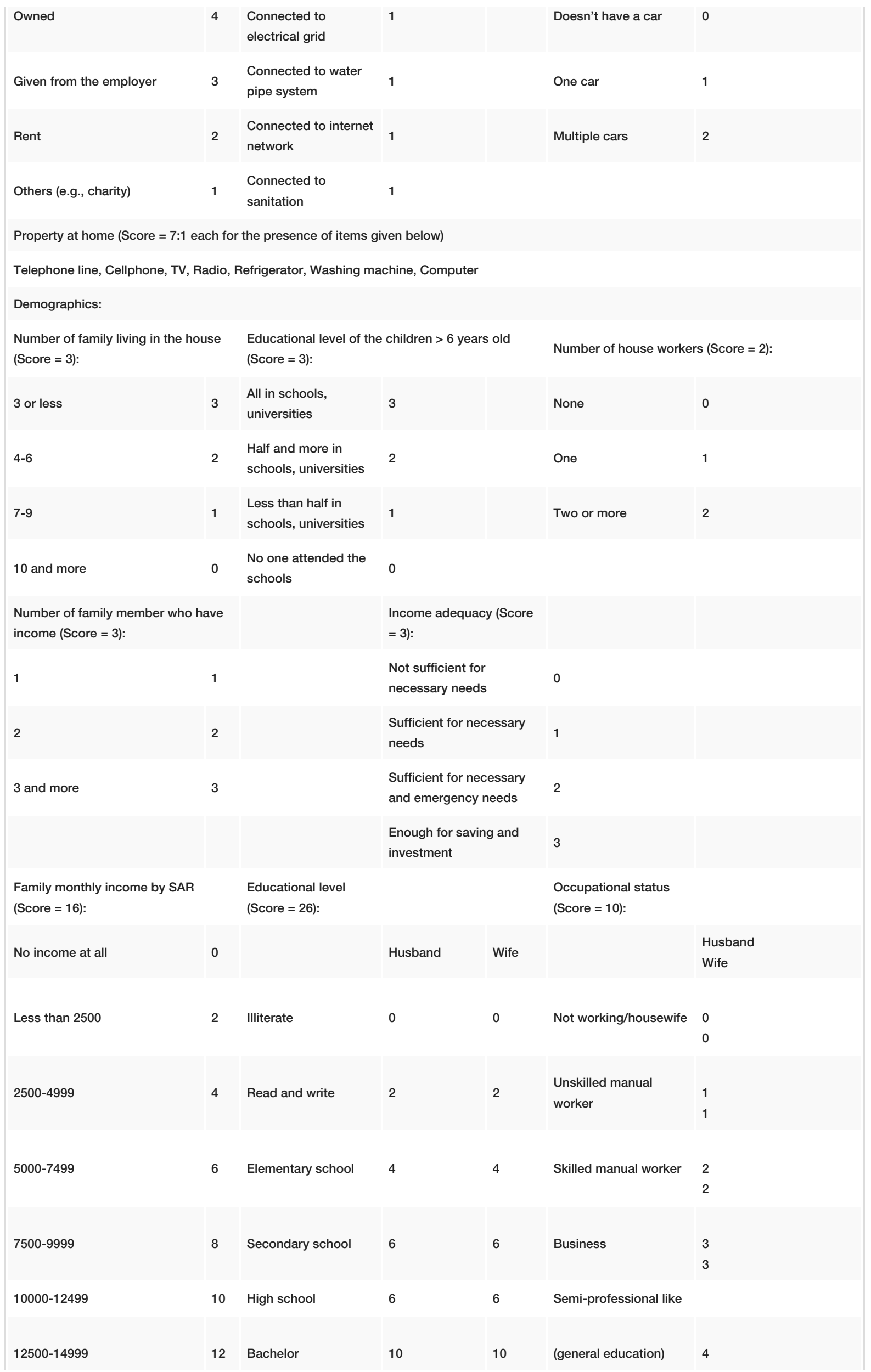




\section{Cureus}

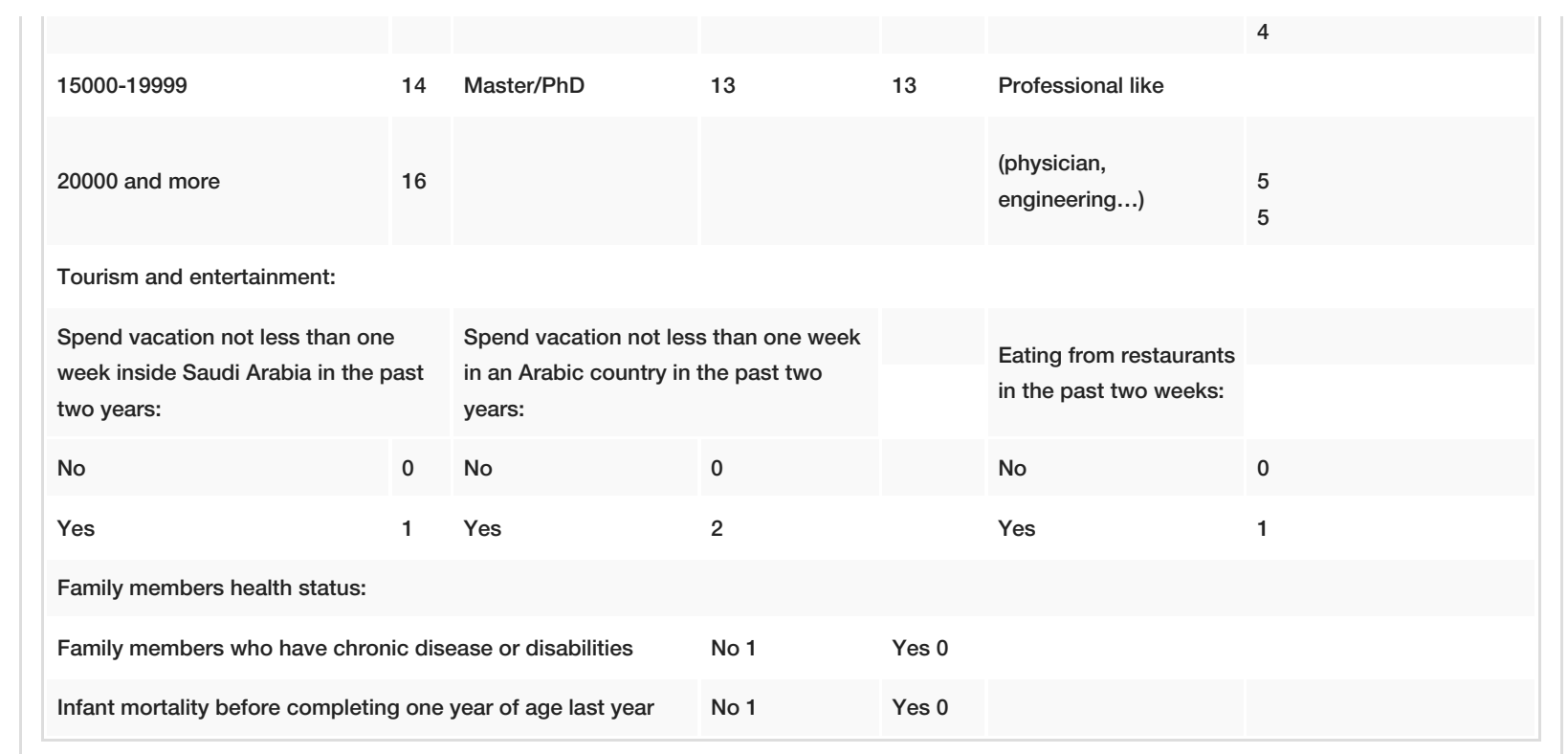

\section{TABLE 3: Elzahrany SES score}

\section{Additional Information \\ Disclosures}

Human subjects: Consent was obtained by all participants in this study. Institutional Review Board (IRB) WR issued approval IRBC/1016/17. Animal subjects: All authors have confirmed that this study did not involve animal subjects or tissue. Conflicts of interest: In compliance with the ICMJE uniform disclosure form, all authors declare the following: Payment/services info: All authors have declared that no financial support was received from any organization for the submitted work. Financial relationships: All authors have declared that they have no financial relationships at present or within the previous three years with any organizations that might have an interest in the submitted work. Intellectual property info: The authors would like to thank Ramzy Elzahrany Ph.D. for sharing the survey of the socioeconomic levels and standards of living in Makkah "Mecca”, Saudi Arabia, Department of Geography, Um Al Qura University, Makkah (Mecca), Saudi Arabia, 2017. Other relationships: All authors have declared that there are no other relationships or activities that could appear to have influenced the submitted work.

\section{Acknowledgements}

The authors would like to thank Ramzy Elzahrany Ph.D. for sharing the survey of the socioeconomic levels and standards of living in Makkah "Mecca", Saudi Arabia. The authors would like to thank Enago (www.enago.com) for the English language review.

\section{References}

1. Blencowe H, Cousens S, Oestergaard MZ, et al.: National, regional, and worldwide estimates of preterm birth rates in the year 2010 with time trends since 1990 for selected countries: a systematic analysis and implications. Lancet. 2012, 379:2162-2172. 10.1016/S0140-6736(12)60820-4

2. Beck S, Wojdyla D, Say L, et al.: The worldwide incidence of preterm birth: a systematic review of maternal mortality and morbidity. Bull World Health Organ. 2010, 88:31-38. 10.2471/BLT.08.062554

3. Children, youth, families and socioeconomic status . (2017). Accessed: July 26, 2017: http://www.apa.org/pi/ses/resources/publications/children-families.aspx.

4. Socioeconomic status. (2017). Accessed: July 27, 2017: http://www.apa.org/topics/socioeconomic-status/.

5. Cantarutti A, Franchi M, Compagnoni MM, Merlino L, Corrao G: Mother's education and the risk of several neonatal outcomes: an evidence from an Italian population-based study. BMC Pregnancy and Childbirth. 2017, 17:221. 10.1186/s12884-017-1418-1

6. Parker JD, Schoendorf KC, Kiely JL: Associations between measures of socioeconomic status and low birth weight, small for gestational age, and premature delivery in the United States. Ann Epidemiol. 1994, 4:2718. 10.1016/1047-2797(94)90082-5

7. Lefmann T, Combs-Orme T, Orme JG: Examining the inter-correlated effects of low income, life stress, and race on birth outcomes: a representative state study. Soc Work Health Care. 2017, 56:450-469. 10.1080/00981389.2017.1316811

8. Mishra D, Singh HP: Kuppuswamy’s socioeconomic status scale-a revision. Indian J Pediatr. 2003, 70:273274. 10.1007/BF02725598

9. Cirino PT, Chin CE, Sevcik RA, Wolf M, Lovett M, Morris RD: Measuring socioeconomic status: reliability and preliminary validity for different approaches. Assessment. 2002, 9:145-55. 10.1177/10791102009002005 


\section{Cureus}

10. Fahmy S, El-Sherbini AF: Determining simple parameters for social classifications for health research. Bull High Inst Public Health. 1983, 13:95-108.

11. El-Gilany A, El-Wehady A, El-Wasify M: Updating and validation of the socioeconomic status scale for health research in Egypt. East Mediterr Health J. 2012, 18:962. 10.26719/2012.18.9.962

12. Campbell EE, Gilliland J, Dworatzek PDN, De Vrijer B, Penava D, Seabrook JA: Socioeconomic status and adverse birth outcomes: a population-based Canadian sample. J Biosoc Sci. 2018, 50:102-113. 10.1017/S0021932017000062

13. Figueroa JF, Frakt AB, Jha AK: Addressing social determinants of health: time for a polysocial risk score [IN PRESS]. JAMA. 2020, 10.1001/jama.2020.2436

14. Hobel CJ, Dunkel-Schetter C, Roesch SC, Castro LC, Arora CP: Maternal plasma corticotropin-releasing hormone associated with stress at 20 weeks' gestation in pregnancies ending in preterm delivery. Am J Obstet Gynecol. 1999, 180:257. 10.1016/s0002-9378(99)70712-x

15. Rich-Edwards JW, Grizzard TA: Psychosocial stress and neuroendocrine mechanisms in preterm delivery . Am J Obstet Gynecol. 2005, 192:S30-S35. 10.1016/j.ajog.2005.01.072 